\title{
Ayurvedic Aspects of Bhallatak w.s.r. Agadtantra
}

\section{${ }^{1}$ Dr. Ravindra R. Pandhare , ${ }^{2}$ Dr. Nagnath S. Gangasagre,${ }^{3}$ Dr. Nilesh M. Salve,${ }^{4}$ Dr. S.Lahankar. ${ }^{5}$ Dr.Sandip Nakhate , ${ }^{6}$ DrVaibhav Bhadage}

\author{
${ }^{1}$ PG scholar department of Agadtantra,Government Ayurvedic College, Osmanabad, Maharashtra, India. \\ ${ }^{2}$ Professor and Head of department, department of Agadtantra, Government Ayurvedic college, Osmanabad, \\ Maharashtra, India. \\ ${ }^{3} \mathrm{PG}$ scholar department of Agadtantra, Government Ayurvedic College, Osmanabad, Maharashtra, India. \\ ${ }^{4}$ Associate professor department of Agadtantra, department of Agadtantra,Government Ayurvedic College, \\ Osmanabad, Maharashtra, India. \\ ${ }^{5} \mathrm{PG}$ scholar department of shalyatantra, Government Ayurvedic College, Osmanabad, Maharashtra, India \\ ${ }^{6} \mathrm{PG}$ scholar department of Rachana sharir, Government Ayurvedic College,Osmanabad, Maharashtra, India.
}

Corresponding Address- Dr. Ravindra R. Pandhare

PG Scholar, Agadtantra Department Govt. Ayurvedic College, Osmanabad, Maharashtra(India) 413501

\begin{abstract}
-
Bhallataka is also known as marking nut tree- dhobi's nut, family belong to anacardiaceae, according to various acharyas bhallatak have various synonyms according to their action. In Ayurveda it is described as vanaspatij visha as in modern science, it is irritant organic vegetable poison. All the parts are poisonous mostly seeds are hazardous to human due to its irritant property. By shodhan sanskara, bhallatak has most beneficial effects. In charak samhita various yogs are explained of bhallatak as a rasayana, the ballataka nut contains bhilwanol and semicarpol as toxic ingredient shows some medicinal property. Bhallataka is also used as non- medico purpose to making a ink or hair dye. Detoxified bhallataka shows some dipan, pachan, bhedana jwaragnha, krumighna, medhya, balya ,vrushya, mutrajanan properties. By its hot potency (Ushna virya) it has some local action like spotjanana. Semicarpol and bhilonwol shows antimicrobial, analgesic, anti-oxidant anti-inflammatory, ant-helmintic activities, this article suggest the ayurvedic review of bhallataka w.s.r. to agadtantra
\end{abstract}

\section{Introduction -}

The marking nut tree belong to anacardiaceae and its fruits called bhilawan, weight about 1.6 to $3.6 \mathrm{~g}$ and has a strong hard, black rind within which is a thick pericarp, it is fleshy pulp of the fruits or seeds, a brownish oily acrid juice which turns black when exposed to air or mixed with lime it is used by dhobis as marking ink for cotton and linen clothes1. By various acharyas different shodhan methods are described in samhitas by purification it is used in various diseases and shows some good effect. Bhallataka grows throughout India in hot weather bhallataka used by externally and internally in both ways. It mentioned as upvisha. The fruit is edible and sweet when ripe, but the black fruit is toxic and it produces a severe allergic reaction, when consumed or its resin comes in contact with the skin2. When proper use of bhallatak it reduces vata and aggravates pitta due to its hot potency, it is very useful in skin disease, loose-motion, piles neuralgia, epilepsy, seeds oil are very effective in rheumatism when apply externally3. The extract obtained from the fruits and nuts is very strong anti-inflammatory, antimicrobial, antioxidant, and anti-carcinogenic properties, It stimulant the central nervous system and hair growth promote, the nuts of semecarpus anacardium show some medicinal uses due to its content various ingredient such as phenolic compounds, biflavonoids, bhilawanols, vitamins, minerals, and amino acid4.

Aim - To study the ayurvedic aspects of bhallatak 
${ }^{1}$ Dr. Ravindra R. Pandhare,International Journal of Ayurvedic \& Herbal Medicine 7(4) July.-Aug. 2017 (2752-2755)

\section{Objective -}

To collect the literature review of bhallattak

To study the systemic effect of bhallatak

To study the toxicity of bhallatak

Bhallatak synonyms-

Sanskrit name - Bhallat, tapan, agni, krumighn, vataril.

-Arushk arushkar, agnik, agnimukhi, bhalli, veervruksha, shophkrut2

-Bhallatak, agni, dahan,tapan, aruskar, anal, krimighnha, tailbeej, vatari, spotbijak,pruthakbeej, dhanurbeej, bhallat, veejpadap, vanhi, vartaru 3.

English name - Marking nut, bhilawan, dhobis nut.

Botanical name - Semecarpus anacardium

Family - Anacardiaceae

Hindi - Bhilawa,

Marathi - Bibba,

Malayalam -- Senkoottaui,

Kannada - Bilawa

Bengali - Bhela

Gujrati $\quad-$ Bhilamo

Punjabi $\quad-$ Fidivittulu

Arabi $\quad-$ Habbulkalb4

Vargikaran -

Ayurvedic - sthavarvish - vanspatij vish

Modern - irritant,organic, vegetable poison

Swarup (Phylotaxy) of bhalltak5 -

Height-25-40 feet in height

Patra (leaves ) - Aaytakar, samuhabadhha, golagra,

Pushpa (flowers) - Eklingi

Fala (fruits) - One inch in length, hrudayakruti(heart shape),chamkile(shini)

Utpatti sthan - Bharat, himalay, bihar, udisa, aassam

Rasayanik sanghatan (chemical composition) -- Bhilawanol

Ayurvedic properties-

Semecarpol.

Guna - Snigdh,laghu,tikshna

Rasa-Katu, tikt, kashay6, 7

Vipak-Madhur 8, katu9.

Virya-Ushna.

Doshkarma-Due to ushna virya and tikshna guna it is kaphavatshamak and pittvardhak

Local action - Sphotjanana, shitprashaman,vishagnhal0.

Internal action $11-$

\begin{tabular}{|c|c|c|}
\hline No. & System & Karma \\
\hline 1 & Pachanvah sansthan (Digestive) & Dipan, pachan, bhedan krumighna \\
\hline 2 & Swasansanshtan (circulatory) & Hrudayuttejit, shothnashan \\
\hline 3 & Mutravah sansthan (excretory) & Mutrajanan(diuretic) \\
\hline 4 & Prajanan sansthan (Reproductive) & Vrushyya, garbhashauttejak \\
\hline 5 & Tapakram (Fever) & Jwaraghn \\
\hline 6 & Twaka & Swedjanam, kushtagnha \\
\hline 7 & Nadisansthan & Medhya, balya \\
\hline
\end{tabular}


${ }^{1}$ Dr. Ravindra R. Pandhare,International Journal of Ayurvedic \& Herbal Medicine 7(4) July.-Aug. 2017 (2752-2755)

\section{Therapeutic uses described in samhita -}

1. Sarpdansh, arshroga, garbhsravak, mashtiskadourbalya, nadidourbalya , apsmar, aamwat pakshaghat grudhrasi ,gulma ,uder ,grahni hrudaydourbalyy ,granthishoth , kas, swas , prameh , shukradourbalya, kusht, vatrakt swhitra jwar ${ }^{12}$.

2. Krumighna, vatjanyrog, udervikar, anaha, prameh, arshrog, kaphvkar, shram shwasrog, vibhandh , shul, ${ }^{13}$.

3. Arsh , vatvikar, firanga, gandmala , krumi, visuchika, gulm, jirn aamvat, kusht, agnimandy, vibandha, rasayan ardit, urusthambha, artavvikar, vajikaran, $\operatorname{charmarog}^{14}$.

Aushadhi matra -- 1-3 ratthi ${ }^{15}$.

Tail - 1-2 drop, awleh- 0.25-0.5 tola, kshirpak-1-2 tola, fal kalk-120-360 mg

Ghatak matra (fatal dose) and ghatak kal (fata periods) - 5 to $10 \mathrm{gm}$ and 12 to 24 hour $^{16}$.

Vishakt lakshane -

1. Dah, vran, mukhshoth, visarp, ${ }^{17}$.

2. Kandu, dah, twakvaivarnya, atisar, jwar, raktmeh, unmad, ${ }^{18}$.

3. Guda-shishna kandu, swedatipravrutti, trushna, alpamutrapravrutti, twakdah-kandu ${ }^{19}$.

Vishakt chikitsa-

1. Til tail, nariyal tail, ghee or ral malahar-local application ${ }^{20}$.

2. Narriyal dugdha, chincha patra swaras, nariyal, lead lotion ${ }^{21}$.

Post mortem appearance --

Blisters in the mouth, throat, mucous membrane in the stomach, congested and inflamed, fatty degeneration of liver ${ }^{22}$.

Medico-legal aspect --

1. Juice of an bhallataka is used as abortificient by means of its application to the os uteri by means of an abortion stick, applied to the genitals for the punishment for adultery, juice has been thrown on the face with an evil intensions. Used by malingerers to produce as artificial bruises, internal administration by quacks - due to accidental poisoning ${ }^{23}$.

2. Apllied externally produces irritaton and blisters containing acrid serum, administration of juice by hakim and vaid due to accidentally. Homicidal poisoning is rare ${ }^{24}$.

\section{Conclusion -}

Bhallataka is very potent herbal plant used as various purposes. It has different synonym according various acharyas. According to its action it has given a various name viz vanhi, krumignh. It belongs to anacardiceae family. By purification it has various system wise effect and various therapeutic uses to treat the hazardous disorder. As per modern science it is organic irritant vegetable poison but according to acharya priyvat sharma it is vishagnha (anti-poisonous) activity. It is used as abortificient in recent time. in that guru snigdha and shit treatment is given, due to it is hot in nature.Its homicidal poisoning is rare. Research and more efforts on Semecarpus anacardium gives us more uses of the plant, mechanism of action and its effectiveness in various disorder.

\section{Refrences -}

1. Modi's medical jurisprudence and toxicology, twenty third edition, edited by Dr.K Mathiharan and Dr Amrit K Patnaik ,Lexis Nexis New Delhi 2006, pg.no-232.

2. https://en.wikipedia.org/wiki/Semecarpus_anacardium cited at 14 may 2017.

3. https://www.bimbima.com/ayurveda/ayurvedic-herb-bhallataka-semecarpus-anacardium/331/ cited at 2017.

4. http://www.valuefood.info/2062/health-benefits-of-bibba-seeds-semecarpus-anacardium/cited at 09 aug 2014. 
${ }^{1}$ Dr. Ravindra R. Pandhare,International Journal of Ayurvedic \& Herbal Medicine 7(4) July.-Aug. 2017 (2752-2755)

5. Rastarngini , pranacharya shri sadanand sharmana virachita, sa cheyam- pandit kashinathshastrin,motilal banarasidas- 11/1979 , pg no 733.

6. Bhavprakash nighantu, padmashri krushnachandra chunekar, swa.dr.gangasahay pandey,choukhambha bharati acadmy -Varanasi.2015 pg.no. -134

7. Rajnighantu of pandit narahari by dr.indradeva triphathi,choukhambha krishnadas academy varanasi ,2010 pg no --352

8. Dravyaguna vidnyan, volume 2 , acharya priyvat sharma choukhambha bharati academy-varanasi, 2005 pg.no.- 166.

9. Dravyaguna vidnyan, volume 2 , acharya priyvat sharma choukhambha bharati academy-varanasi, 2005 pg.no.- 166.

10. Dravyaguna vidnyan, volume 2 , acharya priyvat sharma choukhambha bharati academy-varanasi, 2005 pg.no. -167

11. Rajnighantu of pandit narahari by dr.indradeva triphathi,choukhambha krishnadas academy varanasi ,2010 pg no --353

12. Dravyaguna vidnyan, volume 2 , acharya priyvat sharma choukhambha bharati academy-varanasi, 2005 pg.no.- 167

13. Bhavprakash nighantu, padmashri krushnachandra chunekar, swa.dr.gangasahay pandey,choukhambha bharati acadmy -Varanasi.2015 pg.no. -134

14. Dravyaguna vidnyan, volume 2 , acharya priyvat sharma choukhambha bharati academy-varanasi, 2005 pg.no.-167

15. Dravyaguna vidnyan, volume 2, acharya priyvat sharma choukhambha bharati academy-varanasi, 2005 pg.no. $-167,168$

16. Dravyaguna vidnyan, volume 2 , acharya priyvat sharma choukhambha bharati academy-varanasi, 2005 pg.no.-168

17. Rajnighantu of pandit narahari by dr.indradeva triphathi,choukhambha krishnadas academy varanasi ,2010 pg no --353

18. Bhavprakash nighantu, padmashri krushnachandra chunekar, swa.dr.gangasahay pandey, choukhambha bharati acadmy -Varanasi.2015 pg.no. $-135,136$

19. Rastarngini , pranacharya shri sadanand sharmana virachita, sa cheyam- pandit kashinath shastrin, motilal banarasidas- $11 / 1979$, pg no 733

20. Textbook of forensic medicine and toxicology by anil aggrawal, avichal publishing company , 2016 pg.no. -636

21. Rastarngini , pranacharya shri sadanand sharmana virachita , sa cheyam- pandit kashinathshastrin,motilal banarasidas- 11/1979 , pg no 734

22. Bhavprakash nighantu, padmashri krushnachandra chunekar, swa.dr.gangasahay pandey,choukhambha bharati acadmy -Varanasi.2015 pg.no. - 136

23. Dravyaguna vidnyan, volume 2 , acharya priyvat sharma choukhambha bharati academy-varanasi, 2005 pg.no.-166

24. Dravyaguna vidnyan, volume 2 , acharya priyvat sharma choukhambha bharati academy-varanasi, 2005 pg.no.-166

25. Bhavprakash nighantu, padmashri krushnachandra chunekar, swa.dr.gangasahay pandey,choukhambha bharati acadmy -Varanasi.2015 pg.no. - 136, 137

26. Modi's medical jurisprudence and toxicology, twenty third edition, edited by Dr.K Mathiharan and Dr Amrit K Patnaik ,Lexis Nexis New Delhi 2006, pg.no-23

27. Parikh's textbook of medical jurisprudence Forensic medicine and toxicology sixth ediion by dr C.K. Parikh, CBS publishers \& distributors pvt. Ltd.2010 pg.no. 9.23.

28. modi's medical jurisprudence and toxicology ,twenty third edition, edited by Dr.K Mathiharan and Dr Amrit K Patnaik ,Lexis Nexis New Delhi 2006, pg.no-233-234.. 